\title{
Derivatives of Vibralactone from Cultures of the Basidiomycete Boreostereum vibrans
}

\author{
Meng-Yuan JiAnG,${ }^{a, b}$ Fei WAnG, ${ }^{a}$ Xiao-Long YANG,${ }^{a, b}$ Li-Zhen FAnG,${ }^{a, b}$ Ze-Jun Dong, ${ }^{a}$ \\ Hua-Jie ZHU, ${ }^{a}$ and Ji-Kai LiU*,a \\ ${ }^{a}$ State Key Laboratory of Phytochemistry and Plant Resources in West China, Kunming Institute of Botany, Chinese \\ Academy of Sciences; Kunming 650204, People's Republic of China: and ${ }^{b}$ Graduate School of Chinese Academy of \\ Sciences; Beijing 100049, People's Republic of China. \\ Received May 4, 2008; accepted June 12, 2008; published online June 16, 2008
}

Four new natural products possessing vibralactone skeleton, 1,5-secovibralactone (1), vibralactone B (2), vibralactone C (3) and acetylated vibralactone (4), together with known compound vibralactone (5), had been isolated from cultures of the basidiomycete Boreostereum vibrans. The structures of $1-4$ were elucidated on the basis of spectroscopic methods. The absolute configuration of 1 was suggested to be $S$ by computational methods.

Key words vibralactone; Boreostereum vibrans; basidiomycete

China is extraordinary rich in higher fungi. To date about 10000 species of fungi have been reported from the vast territory of China. Among them, nearly 6000 species, belonging to about 1200 genera, are higher fungi (excluding lichens). Higher fungi in bio-resources belong to the very productive biologically sources which produce a large and diverse variety of secondary metabolites. We have been interested in the biologically active substances present in untapped and diverse source of higher fungi from China. ${ }^{1)}$

The fungus Boreostereum vibrans (BERK. \& M. A. CuRTIS) DAVYDKINA \& BONDARTSEVA (synonym: Stereum vibrans Berk. \& M. A. Curtis) belonged to the family of Boreostereaceae. ${ }^{2)}$ Vibralactone (5), a lipase inhibitor with an unusual fused $\beta$-lactone, was obtained from the culture broth of $B$. vibrans in our previous report. ${ }^{3)}$ Interestingly, a structurally closely related metabolite, percyquinnin, of undetermined relative and absolute configuration has been isolated from cultures of Stereum complicatum. ${ }^{4)}$ Previous chemical investigations on the genus Stereum led to the isolation of various sesquiterpenoids, ${ }^{5-15)}$ acetylenic compounds, ${ }^{16)}$ glycerolipids, ${ }^{17)}$ steroids, ${ }^{18)}$ alkaloids ${ }^{19)}$ benzaldehydes and benzofurans, ${ }^{20,21)}$ phenolic compounds, chromene and aromatic aldehyde derivatives. ${ }^{22,23)}$ To find more compounds with the unique skeleton of vibralactone, the further study was undertaken to investigate the minor constituents of this fungus. Four derivatives of vibralactone: 1,5 -secovibralactone (1), vibralactone B (2), vibralactone C (3) and acetylated vibralactone (4), together with vibralactone (5) were obtained. The isolation and the structural elucidation of new metabolites are described in this report.

The organism was cultured in shakers $(150 \mathrm{rpm})$ with modified (potato-dextrose agar) PDA medium. After culturing for $14 \mathrm{~d}$ at $22^{\circ} \mathrm{C}$, the whole culture broth (211) was filtered and then the filtrate was extracted three times with EtOAc. The crude EtOAc extract $(6 \mathrm{~g})$ was subjected to repeated column chromatography to give pure 1 (46 mg), 2 (9 mg), 3 (14 mg), 4 (7 mg) and 5 (1810 mg).

\section{Results and Discussion}

Compound 1 was obtained as a colorless oil. Its molecular formula was established as $\mathrm{C}_{12} \mathrm{H}_{16} \mathrm{O}_{3}$ by positive HR-ESIMS $\left(m / z 209.1177[\mathrm{M}+\mathrm{H}]^{+}\right.$, Calcd for $\mathrm{C}_{12} \mathrm{H}_{17} \mathrm{O}_{3}$ : 209.1177).
The IR spectrum showed the presence of both carbonyl $\left(1761 \mathrm{~cm}^{-1}\right)$ and hydroxy $\left(3419 \mathrm{~cm}^{-1}\right)$ groups. The ${ }^{1} \mathrm{H}-$ and ${ }^{13} \mathrm{C}-\mathrm{NMR}$ spectral data (Tables 1,2 ) exhibited the signals of two methyls, two methylenes (including one oxygenated), five methines (including four olefinic) and three quaternary carbons (including one carbonyl and two olefinic carbons). The signals at $\delta_{\mathrm{C}} 17.9(\mathrm{q}, \mathrm{C}-11), 25.7$ (q, C-12), 27.8 (t, C8), 120.3 (d, C-9), 134.5 (s, C-10), $\delta_{\mathrm{H}} 1.68(\mathrm{~s}, \mathrm{H}-11), 1.71$ (s, $\mathrm{H}-12), 5.15$ (m, H-9) indicated the presence of an isoprenyl moiety. The remaining moiety contained a carbonyl $\left(\delta_{\mathrm{C}}\right.$ $167.8)$, four olefinic carbons $\left(\delta_{\mathrm{C}} 140.4, \mathrm{~d}, \mathrm{C}-5 ; 138.0, \mathrm{~s}, \mathrm{C}-3\right.$; 123.3 , d, C-2; 112.4, d, C-4), a methine $\left(\delta_{\mathrm{C}} 45.3, \mathrm{~d}, \mathrm{C}-1\right)$ and a hydroxymethyl group $\left(\delta_{\mathrm{C}} 63.8, \mathrm{t}, \mathrm{C}-13\right)$. Comparison of these ${ }^{1} \mathrm{H}$ - and ${ }^{13} \mathrm{C}$-NMR data with those of vibralactone (5), one more double bond was observed in $\mathbf{1}$, suggested that 1 was derived from 5 by ring enlargement. In the ${ }^{1} \mathrm{H}-{ }^{1} \mathrm{H}$ COSY spectra the significant correlations between $\mathrm{H}-1$ and $\mathrm{H}-2, \mathrm{H}-$ 8, $\mathrm{H}-4$ and $\mathrm{H}-5, \mathrm{H}-8$ and $\mathrm{H}-9$ were observed. The gross structure was confirmed by heteronuclear multiple bond connectivity (HMBC) spectrum which showed the key correlations from $\mathrm{H}-1$ to $\mathrm{C}-3$, from $\mathrm{H}-2$ to $\mathrm{C}-4, \mathrm{C}-7$ and $\mathrm{C}-13$ (hydroxymethyl), from $\mathrm{H}-4$ to $\mathrm{C}-2$ and $\mathrm{C}-13$ (Fig. 2). Correlations also observed from $\mathrm{H}-13$ to $\mathrm{C}-2$ and $\mathrm{C}-4$, from $\mathrm{H}-8$ to C-2 and C-7 which suggested that C-13 and the isoprenyl group were attached at $\mathrm{C}-3$ and $\mathrm{C}-1$, respectively.

For the assignment of absolute configuration, the optical rotation values for 1,5-secovibralactone (1) and its enantiomer were calculated. The computational method, B3LYP/aug-cc-pVDZ//B3LYP/6-31G*, for optical rotation calculations was used as our previous research. ${ }^{3)}$ The absolute configuration of $\mathbf{1}$ was identified as $S$ on the basis of the calculating result $+256.7^{\circ}$. The experimental value for optical rotation of $\mathbf{1}$ is $+107.5^{\circ}$. We also proposed a conversion from compound $\mathbf{5}$ to $\mathbf{1}$. Compound $\mathbf{1}$ was the 1,3 sigmatropic $\mathrm{H}$ migration product of $\mathbf{5}$. The migration was facilitated by a "pull" from C-1 initiated by the strain of four-ring and a "push" from the departure of a H-4, providing the seven-member ring with $\mathrm{H}-1$ in $\alpha$-orientation (Chart 1 ). The configuration resembled to the computational method. Thus, 1 was suggested as $(1 S)$-1,5-secovibralactone.

Compound 2 was obtained as colorless crystals. The molecular formula was found to be $\mathrm{C}_{12} \mathrm{H}_{16} \mathrm{O}_{4}$ by HR-ESI-MS 
Table 1. ${ }^{1} \mathrm{H}-\mathrm{NMR}$ Spectroscopic Data $\left(\mathrm{CDCl}_{3}\right)$ for Compounds $\mathbf{1}-\mathbf{5}$

\begin{tabular}{|c|c|c|c|c|c|}
\hline \multirow{2}{*}{ No. } & \multicolumn{5}{|c|}{$\delta_{\mathrm{H}}(J$ in $\mathrm{Hz})$} \\
\hline & $\mathbf{1}^{a)}$ & $2^{a)}$ & $3^{b)}$ & $4^{b)}$ & $5^{a)}$ \\
\hline 1 & $2.55(\mathrm{~m})$ & & & & \\
\hline 2 & $5.46(\mathrm{~d}, 4.4)$ & $3.55(\mathrm{~s})$ & $6.68(\mathrm{brs})$ & $5.65(\mathrm{brs})$ & $5.58(\mathrm{br} \mathrm{s})$ \\
\hline \multirow[t]{2}{*}{4} & $5.91(\mathrm{~d}, 6.8)$ & $2.44(\mathrm{~d}, 16.5)$ & $3.05(\mathrm{~d}, 19.3)$ & $2.78(\mathrm{dd}, 19.2,3.2)$ & $2.76(\mathrm{dd}, 18.9,3.6)$ \\
\hline & & $2.10(\mathrm{dd}, 16.5,6.7)$ & $2.90(\mathrm{dd}, 19.3,5.7)$ & $2.74(\mathrm{dd}, 19.2,2.7)$ & $2.69(\mathrm{~d}, 18.9)$ \\
\hline 5 & $6.59(\mathrm{~d}, 6.8)$ & $4.81(\mathrm{~d}, 6.7)$ & $4.90(\mathrm{~d}, 5.7)$ & $4.80(\mathrm{dd}, 3.2,2.7)$ & $4.78(\mathrm{~d}, 3.6)$ \\
\hline \multirow[t]{2}{*}{8} & $2.68(\mathrm{~m})$ & $2.65(\mathrm{dd}, 15.3,7.2)$ & $2.74(\mathrm{dd}, 15.1,7.3)$ & $2.62(\mathrm{dd}, 15.1,7.3)$ & $2.59(\mathrm{dd}, 15.1,7.3)$ \\
\hline & $2.57(\mathrm{~m})$ & $2.51(\mathrm{dd}, 15.3,7.5)$ & $2.59(\mathrm{dd}, 15.1,7.3)$ & $2.43(\mathrm{dd}, 15.1,7.3)$ & $2.41(\mathrm{dd}, 15.1,7.3)$ \\
\hline 9 & $5.15(\mathrm{~m})$ & $5.15(\mathrm{~m})$ & $5.11(\mathrm{t}, 7.3)$ & $5.11(\mathrm{t}, 7.3)$ & $5.10(t, 7.3)$ \\
\hline 11 & $1.68(\mathrm{~s})$ & $1.66(\mathrm{~s})$ & $1.66(\mathrm{~s})$ & $1.64(\mathrm{~s})$ & $1.62(\mathrm{~s})$ \\
\hline 12 & $1.71(\mathrm{~s})$ & $1.74(\mathrm{~s})$ & $1.74(\mathrm{~s})$ & $1.73(\mathrm{~s})$ & $1.71(\mathrm{~s})$ \\
\hline \multirow[t]{2}{*}{13} & $4.21(\mathrm{~d}, 13.5)$ & $3.92(\mathrm{~d}, 12.8)$ & $9.83(\mathrm{~s})$ & $4.66(\mathrm{brs})$ & $4.20(\mathrm{br} \mathrm{s})$ \\
\hline & $4.17(\mathrm{~d}, 13.5)$ & $3.82(\mathrm{~d}, 12.8)$ & & & \\
\hline$\underline{\mathrm{MeCO}}$ & & & & $2.11(\mathrm{~s})$ & \\
\hline
\end{tabular}

a) Data recorded by Bruker AM- 400 and $b$ ) by DRX-500 spectrometers.

Table 2. ${ }^{13} \mathrm{C}$-NMR Spectroscopic Data $\left(\mathrm{CDCl}_{3}\right)$ for Compounds $\mathbf{1}-\mathbf{5}$

\begin{tabular}{rrrrrr}
\hline \hline & \multicolumn{5}{c}{$\delta_{\mathrm{C}}(\mathrm{ppm}), \mathrm{mult}}$. \\
\cline { 2 - 6 } No. & \multicolumn{6}{c}{$\mathbf{1}^{b)}$} & \multicolumn{2}{c}{$\mathbf{2}^{a)}$} & $\mathbf{3}^{b)}$ & \multicolumn{1}{c}{$\mathbf{4}^{b)}$} & \multicolumn{1}{c}{$\mathbf{5}^{b)}$} \\
\hline 1 & $45.3, \mathrm{~d}$ & $69.0, \mathrm{~s}$ & $76.1, \mathrm{~s}$ & $75.4, \mathrm{~s}$ & $75.0, \mathrm{~s}$ \\
2 & $123.3, \mathrm{~d}$ & $61.3, \mathrm{~d}$ & $144.6, \mathrm{~d}$ & $125.1, \mathrm{~d}$ & $122.2, \mathrm{~d}$ \\
3 & $138.0, \mathrm{~s}$ & $77.1, \mathrm{~s}$ & $146.8, \mathrm{~s}$ & $141.3, \mathrm{~s}$ & $146.7, \mathrm{~s}$ \\
4 & $112.4, \mathrm{~d}$ & $31.7, \mathrm{t}$ & $34.1, \mathrm{t}$ & $37.7, \mathrm{t}$ & $37.2, \mathrm{t}$ \\
5 & $140.4, \mathrm{~d}$ & $82.3, \mathrm{~d}$ & $78.0, \mathrm{~d}$ & $78.2, \mathrm{~d}$ & $78.6, \mathrm{~s}$ \\
7 & $167.8, \mathrm{~s}$ & $168.5, \mathrm{~s}$ & $170.0, \mathrm{~s}$ & $172.4, \mathrm{~s}$ & $173.2, \mathrm{~s}$ \\
8 & $27.8, \mathrm{t}$ & $25.9, \mathrm{t}$ & $27.3, \mathrm{t}$ & $27.5, \mathrm{t}$ & $27.5, \mathrm{t}$ \\
9 & $120.3, \mathrm{~d}$ & $116.2, \mathrm{~d}$ & $116.2, \mathrm{~d}$ & $117.1, \mathrm{~d}$ & $117.2, \mathrm{~d}$ \\
10 & $134.5, \mathrm{~s}$ & $137.0, \mathrm{~s}$ & $137.2, \mathrm{~s}$ & $136.2, \mathrm{~s}$ & $135.9, \mathrm{~d}$ \\
11 & $17.9, \mathrm{q}$ & $18.0, \mathrm{q}$ & $18.0, \mathrm{q}$ & $18.0, \mathrm{q}$ & $17.9, \mathrm{q}$ \\
12 & $25.7, \mathrm{q}$ & $25.8, \mathrm{q}$ & $25.8, \mathrm{q}$ & $25.8, \mathrm{q}$ & $25.7, \mathrm{q}$ \\
13 & $63.8, \mathrm{t}$ & $60.7, \mathrm{t}$ & $188.9, \mathrm{~d}$ & $62.0, \mathrm{t}$ & $61.1, \mathrm{t}$ \\
$\mathrm{CH}_{3} \mathrm{CO}$ & & & & $170.5, \mathrm{~s}$ & \\
$\mathrm{CH}_{3} \mathrm{CO}$ & & & & $20.8, \mathrm{q}$ & \\
\hline
\end{tabular}

a) Data recorded by Bruker AM- -400 and $b$ ) by DRX-500 spectrometers.

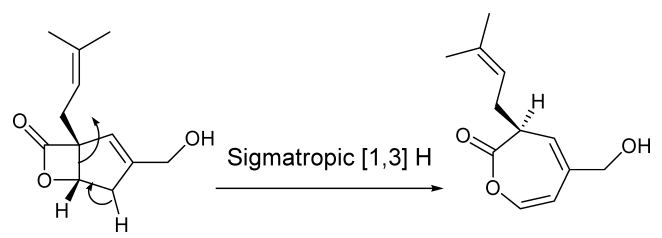

Chart 1. Conversion from $\mathbf{5}$ to $\mathbf{1}$

$\left(\mathrm{m} / z 247.0953[\mathrm{M}+\mathrm{Na}]^{+}\right.$, Calcd for $\mathrm{C}_{12} \mathrm{H}_{16} \mathrm{O}_{4} \mathrm{Na}$ : 247.0946). Its IR spectrum showed the presence of a carbonyl (1811 $\left.\mathrm{cm}^{-1}\right)$ and a double bond $\left(1631 \mathrm{~cm}^{-1}\right)$, respectively. The ${ }^{1} \mathrm{H}-$ and ${ }^{13} \mathrm{C}$-NMR spectral data of $\mathbf{2}$ (Tables 1,2 ) were similar to those of 5, which suggested that $\mathbf{2}$ possessed the same vibralactone skeleton. The characteristic differences were that $\delta_{\mathrm{C}}$ for carbons 2 and 3 in $\mathbf{2}\left(\delta_{\mathrm{C}} 61.3,77.1\right.$, respectively) were shifted upfield compared to those of $\mathbf{5}\left(\delta_{\mathrm{C}} 122.2,146.7\right.$, respectively). The differences were caused by the double bond in 5 being displaced by an epoxy ring in $\mathbf{2}$. In the HMBC spectrum of $\mathbf{2}$ the expected significant correlations: from $\mathrm{H}-2$ to C-4, C-5, C-7, C-8 and C-13 were observed. The relative configurations of 2 were established by rotating frame Over-

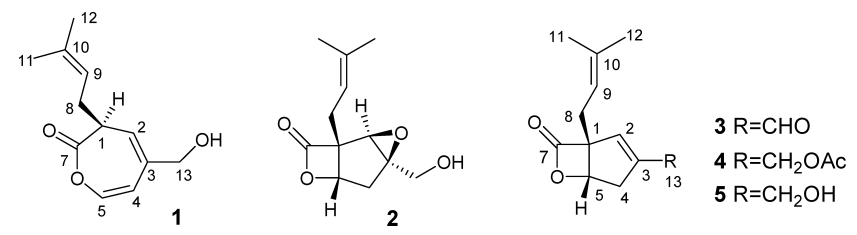

Fig. 1. Structures of $\mathbf{1}-\mathbf{5}$

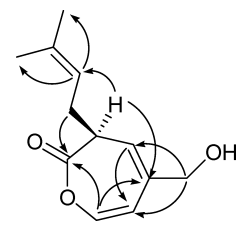

Fig. 2. Selected HMBC Correlations of $\mathbf{1}$

hauser enhancement spectroscopy (ROESY) experiment. The correlations between $\mathrm{H}-2$ and $\mathrm{H}-13$ reflected that the epoxide ring was $\beta$-oriented. From the above data, compound $\mathbf{2}$ was identified as $\left(1 S, 2 R^{*}, 3 R^{*}, 5 S\right)$-2,3-epoxyvibralactone and named vibralactone $B$.

Compound 3 was obtained as a colorless oil. The molecular formula of $\mathrm{C}_{12} \mathrm{H}_{14} \mathrm{O}_{3}$ was established by HR-ESI-MS $(\mathrm{m} / \mathrm{z}$ $229.0840[\mathrm{M}+\mathrm{Na}]^{+}$, Calcd for $\mathrm{C}_{12} \mathrm{H}_{14} \mathrm{O}_{3} \mathrm{Na}: 229.0840$ ). The IR spectrum showed a $\beta$-lactone and a conjugated carbonyl group absorption bands at 1822 and $1686 \mathrm{~cm}^{-1}$, respectively. The ${ }^{1} \mathrm{H}$ - and ${ }^{13} \mathrm{C}$-NMR spectral data (Tables 1,2 ) of $\mathbf{3}$ were very similar to that of $5^{22)}$ The main difference was the hydroxymethyl group in 5 replaced by aldehyde $\left(\delta_{\mathrm{C}} 188.9, \delta_{\mathrm{H}}\right.$ 9.83 ) in 3. Based on the NMR data and the reference, ${ }^{24)}$ the structure of 3 was suggested as shown in Fig. 1 and named vibralactone $\mathrm{C}$.

The molecular formula of $\mathbf{4}$ was deduced as $\mathrm{C}_{14} \mathrm{H}_{18} \mathrm{O}_{4}$ from its HR-ESI-MS. Comparison of the ${ }^{1} \mathrm{H}-$ and ${ }^{13} \mathrm{C}-\mathrm{NMR}$ spectra data of $\mathbf{4}$ with $\mathbf{5}$ revealed that $\mathbf{4}$ had one more acetyl group $\left(\delta_{\mathrm{C}} 170.5,20.8, \delta_{\mathrm{H}} 2.11\right)$ than $\mathbf{5}$, which caused downfield chemical shift of $\mathrm{H}-13\left(\delta_{\mathrm{H}} 4.66\right)$. Thus, the structure of compound 4 was established as shown in Fig. 1 and named acetylated vibralactone. 


\section{Experimental}

General Experimental Procedures Melting point was measured on an XRC-1 apparatus (Sichuan University, Sichuan, People's Republic of China) Optical rotations were obtained in Horiba SEPA-300 digital polarimeter. UV spectra were measured in Shimadzu UV-2401 PC spectrophotometer. IR spectra were recorded on Bruker Tensor-27 spectrometer. NMR spectra were performed on Bruker AM-400 and DRX-500 instruments in $\mathrm{CDCl}_{3}$ with TMS as internal standard. Mass spectra were recorded on VG Autospec3000 mass spectrometer and API QSTAR pulsar 1 spectrometer. Column chromatography (CC) and TLC were carried out on Silica gel (200-300 mesh) and precoated silica gel $\mathrm{GF}_{254}$ plates (Qingdao Marine Chemical Inc. China).

Mushroom Material and Culture B. vibrans was provided and fermented by Prof. Da-Gan Ji, Kunming Institute of Botany. The voucher specimen was deposited at the Herbarium of Kunming Institute of Botany, Chinese Academy of Sciences. Culture medium: potato (peeled) $200.0 \mathrm{~g}$, glucose $20.0 \mathrm{~g}, \mathrm{KH}_{2} \mathrm{PO}_{4} 3.0 \mathrm{~g}, \mathrm{MgSO}_{4} 1.5 \mathrm{~g}$, citric acid $0.1 \mathrm{~g}$, and thiamin hydrochloride $10 \mathrm{mg}$ in 11 of deionized water ( $\mathrm{pH} 6.5$ before autoclaving). The fungus was grown in Erlenmeyer flasks (size: $500 \mathrm{ml}$; media: $300 \mathrm{ml}$ ). Fermentation was carried out on a rotary shaker at $22^{\circ} \mathrm{C}$ and $150 \mathrm{rpm}$ for $14 \mathrm{~d}$.

Extraction and Isolation The mycelium from 211 was filtered, and the filtrate was extracted three times with EtOAc (201). The organic layer was concentrated under a reduced pressure to give a crude extract $(6.0 \mathrm{~g})$. The residue was subjected to column chromatography over silica gel $(4 \times 40 \mathrm{~cm}$, 200 -300 mesh, 45 g) eluting with petroleum ether/EtOAc $(20: 1,15: 1$, $9: 1,7: 1,5: 1, \mathrm{v} / \mathrm{v}$; each 1.21 ) to afford eleven fractions: fractions $\mathrm{A}-\mathrm{D}$ (petroleum ether/EtOAc, 20:1), fractions E, F (petroleum ether/EtOAc, $15: 1$ ), fractions $\mathrm{G}, \mathrm{H}$ (petroleum ether/EtOAc, 9:1), fraction I (petroleum ether/EtOAc, $7: 1$ ) and fraction $\mathrm{K}$ (petroleum ether/EtOAc, $5: 1$ ). Fraction D $(100 \mathrm{mg})$ was rechromatographed on silica gel column $(2 \times 30 \mathrm{~cm}, 200-300$ mesh, $9 \mathrm{~g})$ eluting with petroleum ether/EtOAc $(30: 1, \mathrm{v} / \mathrm{v}, 0.31)$ to obtain $24 \mathrm{mg}$ mixture, which was further purified on silica gel column eluting with petroleum ether to afford $3(14 \mathrm{mg})$ and $4(7 \mathrm{mg})$. Fraction $\mathrm{F}(135 \mathrm{mg})$ was purified on silica gel column $(2 \times 30 \mathrm{~cm}, 200-300$ mesh, $9 \mathrm{~g})$ eluting with petroleum ether/EtOAc $(10: 1,0.41)$ to give $1(46 \mathrm{mg})$. Fraction $\mathrm{H}(2.3 \mathrm{~g})$ was rechromatographed on silica gel column $(3 \times 30 \mathrm{~cm}, 200-300 \mathrm{mesh}$, $25 \mathrm{~g})$ to afford $\mathbf{5}(1810 \mathrm{mg})$. The fraction $\mathrm{K}(112 \mathrm{mg})$ was further purified by repeated chromatographies on silica gel column $(2 \times 30 \mathrm{~cm}, 200-300 \mathrm{mesh}$, $9 \mathrm{~g})$ eluting with petroleum ether/EtOAc $(7: 1 ; 0.41)$ to yield $2(9 \mathrm{mg})$.

1,5-Secovibralactone (1): Colorless oil. $[\alpha]_{\mathrm{D}}^{14}+107.5^{\circ}\left(c=0.34, \mathrm{CHCl}_{3}\right)$. UV $\lambda_{\text {max }}\left(\mathrm{CHCl}_{3}\right) \mathrm{nm}(\log \varepsilon): 240(2.69), 220$ (2.17). IR (film) $\mathrm{cm}^{-1}: 3419$, 2967, 2920, 2861, 1761, 1648, 1605, 1450, 1378, 1264, 1112. NMR data, see Tables 1 and 2. Positive HR-ESI-MS $m / z: 209.1177[\mathrm{M}+\mathrm{H}]^{+}(\mathrm{Calcd}$ for $\left.\mathrm{C}_{12} \mathrm{H}_{17} \mathrm{O}_{3}: 209.1177\right)$

Vibralactone B (2): Colorless needles (EtOAc). mp $115-116^{\circ} \mathrm{C} .[\alpha]_{\mathrm{D}}^{24}$ $-94.4^{\circ}\left(c=0.02, \mathrm{CHCl}_{3}\right)$. IR $(\mathrm{KBr}) \mathrm{cm}^{-1}: 3428,2929,2858,1811,1631$ 1450, 1319, 1131, 1015. NMR data, see Tables 1 and 2. Positive FAB-MS $m / z: 225[\mathrm{M}+\mathrm{H}]^{+}, 317[\mathrm{M}+\mathrm{Gly}+\mathrm{H}]^{+}, 409[\mathrm{M}+2 \mathrm{Gly}+\mathrm{H}]^{+}$. Positive HRESI-MS $m / z$ : $247.0953[\mathrm{M}+\mathrm{Na}]^{+}$(Calcd for $\mathrm{C}_{12} \mathrm{H}_{16} \mathrm{O}_{4} \mathrm{Na}$ : 247.0946).

Vibralactone C (3): Colorless oil; $[\alpha]_{\mathrm{D}}^{17}-288.9^{\circ}\left(c=0.08, \mathrm{CHCl}_{3}\right)$. UV $\lambda_{\max }\left(\mathrm{CHCl}_{3}\right) \mathrm{nm}(\log \varepsilon): 245$ (4.13). IR (film) $\mathrm{cm}^{-1}: 2969,2921,2857$, $2728,1822,1686,1613,1422,1380,1108,1004,833$. NMR data, see Tables 1 and 2. Positive HR-ESI-MS $m / z$ : $229.0840[\mathrm{M}+\mathrm{Na}]^{+}$(Calcd for $\mathrm{C}_{12} \mathrm{H}_{14} \mathrm{O}_{3} \mathrm{Na}: 229.0840$ ).
Acetylated Vibralactone (4): Colorless oil; $[\alpha]_{\mathrm{D}}^{17}-89.2^{\circ} \quad(c=0.19$, $\mathrm{CHCl}_{3}$ ). IR (KBr) cm ${ }^{-1}: 2967,2920,2857,1822,1747,1443,1378,1241$, $1111,1008,833$. NMR data, see Tables 1 and 2. Positive HR-ESI-MS $m / z$ : $273.1104[\mathrm{M}+\mathrm{Na}]^{+}$(Calcd for $\left.\mathrm{C}_{14} \mathrm{H}_{18} \mathrm{O}_{4} \mathrm{Na}: 273.1102\right)$.

Acknowledgment This work was supported by the National Nature Science Foundation of China (KSCX1-YW-R-24; KSCX2-YW-G-025) and National Natural Science Foundation of China (30671385).

\section{References and Notes}

1) Liu J. K., Heterocycles, 57, 157-167 (2002).

2) Davydkina M. A., Bondartseva T. A., Novitates Systematicae Plantarum non Vascularium, 13, 74-76 (1976).

3) Liu D. Z., Wang F., Liao T. G., Tang J. G., Steglish W., Zhu H. J., Liu J. K., Org. Lett., 8, 5749-5752 (2006).

4) Hopmann C., Kurz M., Mueller G., Toti L., EP 1142886 (2001).

5) Xie J. L., Li L. P., Dai Z. Q., J. Org. Chem., 57, 2313-2316 (1992).

6) Ayer W. A., Saeedi-Ghomi M. H., Can. J. Chem., 59, 2536-2538 (1981).

7) Mellows G., Mantle P. G., Feline T. C., Williams D. J., Phytochemistry, 12, 2717-2720 (1973).

8) Ayer W. A., Saeedi-Ghomi M. H., Tetrahedron Lett., 22, 2071-2074 (1981).

9) Yun B. S., Lee I. K., Cho Y., Cho S. M., Yoo I. D., J. Nat. Prod., 65 $786-788(2002)$

10) Li G. H., Duan M., Yu Z. F., Li L., Dong J. Y., Wang X. B., Guo J. W., Huang R., Wang M., Zhang K. Q., Phytochemistry, 69, 1439-1445 (2008).

11) Mantle P. G., Mellows G., Trans. Br. Mycol. Soc., 61, 513-519 (1973).

12) Yoo N. H., Kim J. P., Yun B. S., Ryoo I. J., Lee I. K., Yoon E. S., Koshino H., Yoo I. D., J. Antibiot., 59, 110-113 (2006).

13) Kim Y. H., Yun B. Y., Ryoo I. J., Kim J. P., Koshino H., Yoo I. D., J. Antibiot., 59, 432-434 (2006).

14) Xie J. L., Li L. P., Chin. J. Chem., 10, 537-543 (1992).

15) Li G. H., Li L., Duan M., Zhang K. Q., Chem. Biodiv., 3, 210-216 (2006).

16) Dubin G. M., Fkyerat A., Tabacchi R., Phytochemistry, 53, 571—574 (2000).

17) Doljak B., Cateni F., Anderluh M., Procida G., Zilic J., Zacchigna M., Drug Dev. Ind. Pharm., 32, 635-643 (2006).

18) Cateni F., Doljak B., Zacchigna M., Anderluh M., Piltaver A., Scialino G., Banfi E., Bioorg. Med. Chem. Lett., 17, 6330 -6334 (2007).

19) Ito-Kobayashi M., Aoyagi A., Tanaka I., Muramatsu Y., Umetani M., Takatsu T., J. Antibiot., 61, 128-135 (2008).

20) Nair M. S. R., Anchel M., Phytochemistry, 16, 390-392 (1977).

21) Bu'Lock J. D., Kaye B., Hudson A. T., Phytochemistry, 10, 1037 1046 (1971).

22) Omolo J. O., Anke H., Sterner O., Phytochemistry, 60, 431-435 (2002).

23) Yun B. S., Cho Y., Lee I. K., Cho S. M., Lee T. H., Yoo I. D., J. Antibiot., 55, 208-210 (2002).

24) Zhou Q., Snider B. B., Org. Lett., 10, 1401-1404 (2008). 